\title{
Programas de responsabilidad social externa del Banco Occidental de Descuento
}

\author{
External social responsibility programs of the Western Discount Bank
}

Programas de responsabilidade social externa do Western Discount Bank

Recibido: mayo 2019

Arbitrado: junio 2019

Publicado: septiembre 2019
《Lilibeth Gutiérrez

lilygutierrez2408@hotmail.com ORCID: 0000-0002-2189-5064

Universidad Nacional Experimental Rafael María Baralt, Venezuela

\section{RESUMEN}

El propósito de la investigación fue identificar los programas de responsabilidad social externa presentes en las operaciones del banco occidental de descuento del municipio LagunillasVenezuela. Se diseñó una investigación descriptiva, con diseño no experimental, de campo y transeccional. La población quedó representada por veintisiete (27) sujetos seleccionados que ocupan los cargos de gerentes, subgerentes y ejecutivos de ventas. Como técnica de recolección de datos se usó la encuesta, a través de un cuestionario de 8 ítems con escala de frecuencia. Validado por el juicio de expertos y la confiabilidad medida por el coeficiente Alpha de Crombach, cuyo valor fue de 0,913. Para el análisis de los datos se utilizó la estadística descriptiva a través del cálculo de la media aritmética. Se concluye, que los programas de RSE poseen alta presencia en las operaciones de la banca analizada y los enfoques más comunes son en desarrollo social y desarrollo económico.

Palabras clave: Desarrollo económico; desarrollo social; operaciones; programas, responsabilidad social externa

ABSTRACT

RESUMO

The purpose of the research was to identify the external social responsibility programs present in the operations of the western discount bank of the municipality of Lagunillas-Venezuela. A descriptive research was designed, with a nonexperimental, field and transectional design. The population was represented by twenty-seven (27) selected subjects who occupy the positions of managers, assistant managers and sales executives. As a data collection technique, the survey was used, through an 8-item questionnaire with a frequency scale. Validated by expert judgment and the reliability measured by Crombach's Alpha coefficient, whose value was 0.913. Descriptive statistics were used for data analysis through the calculation of the arithmetic mean. It is concluded that CSR programs have a high presence in the operations of the bank analyzed and the most common approaches are in social development and economic development.

Key words: Economic development; social development; operations; programs, external social responsibility
O objetivo da pesquisa foi identificar os programas de responsabilidade social externa presentes nas operações do banco ocidental de descontos do município de Lagunillas-Venezuela. Foi elaborada uma pesquisa descritiva, com desenho não experimental, de campo e transversal. A população foi representada por vinte e sete (27) sujeitos selecionados que ocupam os cargos de gerentes, gerentes adjuntos e executivos de vendas. Como técnica de coleta de dados, utilizou-se a pesquisa, por meio de um questionário de 8 itens com escala de frequência. Validado por julgamento de especialistas e a confiabilidade medida pelo coeficiente Alfa de Crombach, cujo valor foi de 0,913. A estatística descritiva foi utilizada para a análise dos dados por meio do cálculo da média aritmética. Conclui-se que os programas de RSE têm grande presença nas operações do banco analisado e as abordagens mais comuns estão no desenvolvimento social e no desenvolvimento econômico.

Palavras chave: Desenvolvimento econômico; desenvolvimento social; operações; programas, responsabilidade social externa 


\section{INTRODUCCIÓN}

$\mathrm{H}$ oy día, la responsabilidad social de las empresas está cada vez más presente en distintos ámbitos del mundo económico, social, político y académico, lo que ha llevado a desarrollar iniciativas públicas, empresariales, sindicales y sociales importantes en este campo. Es así, como en la actualidad se habla sobre el tema de responsabilidad social, tanto a nivel nacional como internacional.

Actualmente en Venezuela y desde el año 2000, se ha venido impulsando la promoción en el sector empresarial privado del compromiso con la responsabilidad social y la inversión social para lograr el desarrollo sustentable, es decir, satisfacer necesidades del presente sin comprometer los recursos de generaciones futuras.

En este sentido, la responsabilidad social se presenta como una estrategia que implica el compromiso de las empresas, a través de la aplicación sistemática de recursos, para respetar y promover los derechos de las personas, el crecimiento de la sociedad y el cuidado del ambiente (Añez y otros,2008).

Este compromiso se traduce en acciones concretas que buscan el beneficio de todos los actores involucrados en las actividades de la empresa (accionistas, trabajadores, proveedores, distribuidores y la comunidad en su conjunto) alcanzando un mejor desempeño y logrando su sostenibilidad y la de su entorno; todo ello como soporte de los elementos que conforman la responsabilidad social empresarial.

En el marco de referencia, en el ámbito de la responsabilidad social empresarial, se distinguen dos (2) vertientes o dimensiones, una interna (RSI) y otra externa (RSE). En la investigación, se planteó como objetivo identificar los programas de responsabilidad social externa presentes en las operaciones del banco occidental de descuento del municipio Lagunillas-Venezuela.

Al respecto, se aclara, los bancos universales específicamente en el estado Zulia, han evolucionado en los últimos años hacia modelos organizacionales cuya estructura está orientada a fomentar la generación de una organización socialmente responsables, donde impere el desafío a propiciar procesos, que promuevan nuevas formas de hacer las cosas, pero con mira a establecer trabajo en equipo con un alto desempeño.

Es así, como el Banco Occidental de Descuento considerada como la institución principal del estado Zulia, con 55 años de experiencia y cuya sede principal se encuentra ubicada en Maracaibo, pero con otras sedes de igual importancia tal como es el caso de las oficinas ubicadas en el municipio Lagunillas; han implementado dentro de sus políticas gerenciales, estrategias de manera que se estimule un cambio en su comportamiento, hacia más estrategias positivas, que les permita obtener una ventaja competitiva en los mercados financieros globalizados.

Con base a lo descrito, el objeto de estudio se centra en la responsabilidad social externa en las operaciones del banco occidental de descuento del municipio Lagunillas-Venezuela. Para lograr 
este objetivo, se analizaron los programas de RSE presentes en las operaciones de estos bancos, a través de los programas de desarrollo económico y desarrollo social.

\section{Responsabilidad social externa}

De acuerdo, a la Comunidad Económica Europea (2001), el ámbito externo de la responsabilidad social empresarial constituye una dimensión de acción e inversión para la empresa en la búsqueda de mejorar la calidad de vida de los menos favorecidos, protección al medio ambiente, oportunidades de empleo, programas de educación y capacitación, participación en problemas locales del entorno, programas de viviendas, de centros de cuidado infantil, actividades recreativas, entre otras. Se trata de hacer de la empresa un ente responsable de las necesidades que aquejan a su entorno, y se relacionan con una responsabilidad político-social, en cumplimiento de sus deberes para con el mismo.

Por su parte, Navarro (2012) considera que, la primera responsabilidad de una empresa con la sociedad es cumplir con su misión económica de satisfacer tanto las necesidades como expectativas de sus clientes, a través de la producción de bienes y servicios, aunque recordando que la riqueza es de origen, naturaleza y finalidad social. Sin embargo, en el mismo momento de su formación y localización, asume un conjunto de responsabilidades.

A su vez, Navarro (2012), señala que, la empresa tiene la responsabilidad de cooperar en la solución de problemas que le atañen como miembro de la comunidad donde opera, pues no hay manera de abstraerse del espacio y tiempo donde se inserta. La realidad obliga a tomar conciencia no sólo del medio ambiente, sino también de problemas con los cuales coexiste: pobreza, inseguridad, salud, educación, entre otros.

Así, la empresa depende de la salud, estabilidad y prosperidad de las comunidades donde opera. Por otro lado, afirma este autor, la reputación de una empresa en su lugar de ubicación, su imagen como empresario y productor, también como agente de la vida local, influye sin duda en su competitividad, así como en la comunidad presente.

Bajo este enfoque, Gómez (2013) considera, la responsabilidad social externa de la empresa es, en esencia, un concepto con arreglo, al cual ésta decide voluntariamente contribuir al logro de una sociedad mejor y medio ambiente más limpio. En un momento en el que, la globalización de los mercados y la competitividad entre empresas se hace más evidente, un número creciente de ellas están reconociendo cada vez más claramente su responsabilidad social externa y la consideran parte de su identidad.

Esta responsabilidad, asevera Martínez (2011), se debe expresar frente a todos los interlocutores de la empresa, que pueden a su vez influir en su éxito. Esta evolución refleja las mayores expectativas que despierta en los ciudadanos y en todos los interesados la transformación del papel de las empresas en la nueva sociedad cambiante de hoy en día. 
Ahora bien, los autores Gómez (2013) y Martínez (2011), coinciden cuando definen la dimensión externa de la responsabilidad social, como las relaciones de la empresa con interlocutores externos y entorno social: clientes, proveedores, comunidades locales, administración pública, organizaciones no gubernamentales, las entidades de interés social, consumidores. Así, según estos autores, la empresa tiene una responsabilidad social externa ante los siguientes grupos de interés: consumidores, proveedores, comunidad, competidores, gobierno, y medio ambiente.

En este sentido, Fernández (2009), indica que, como parte de su responsabilidad social externa, la empresa debe ofrecer de manera eficaz, ética y ecológica productos y servicios que los consumidores necesitan, buscando establecer relaciones duraderas con sus clientes, centrando toda su organización en la comprensión de lo que desean; ofreciéndoles calidad, seguridad, fiabilidad y servicios superiores, para obtener mayores beneficios. A tal efecto, indica este autor, la responsabilidad social externa se define como la obligación de una empresa de optimizar su efecto positivo y minimizar su efecto negativo en la sociedad. Al respecto, considera, son cuatro (4) los ámbitos de la RSE: económica, legal, ética y voluntaria.

Por otro lado, las prácticas de la empresa socialmente responsable, según lo acotan Bultera y Díaz (2012), reflejan el compromiso a hacer negocios de forma que preserve o aumente, en lugar de dañar, beneficios a las comunidades cercanas, trabajadores, medio ambiente y conducta económica justa. Aunque no basta con el sólo compromiso, este proceso debe ir acompañado de mecanismos claros de implementación o puesta en práctica de los principios y valores de la responsabilidad social, a través de los programas de responsabilidad externa, de desarrollo social y económico.

Cabe considerar que el conjunto de planteamientos expuestos por los autores citados, generan condiciones para el desarrollo social y económico, así como los grupos de interés y ámbitos en donde se desarrollan las acciones de RSE, constituyendo espacios abiertos en la inversión social, entendida como aquellos gastos que hace la empresa en labores de responsabilidad social en su entorno a través de sus políticas. De este modo, la RSE, en las operaciones del banco occidental de descuento del municipio Lagunillas-Venezuela., se refiere a la preocupación de éstas por el desarrollo de la sociedad, mejora de la calidad de vida y seguridad social, esto es sentir preocupación por la sociedad, siendo así socialmente responsable.

\section{Programas de la responsabilidad social externa}

Las prácticas de la empresa socialmente responsable, según Baltera y Díaz (2012), reflejan el compromiso a hacer negocios de forma que preserve o aumente, en lugar de dañar, beneficios a las comunidades cercanas, trabajadores, medio ambiente y la conducta económica justa. Sin

embargo, no basta con el sólo compromiso, este proceso debe ir acompañado de mecanismos claros de implementación o puesta en práctica de los principios y valores de la responsabilidad 
social, a través de programas de responsabilidad externa, incluyendo seguimiento y verificación independientes.

Estos tipos de programas podrían ser realizados de forma particular por una empresa, según Gómez (2013), disponiendo de sus propios recursos y de sus propios métodos. Otras empresas lo hacen de forma combinada, usando sus recursos y apoyándose en instituciones gubernamentales y en las asociaciones comunitarias (juntas de vecinos, clubes, asociaciones, entre otras). Por lo regular, los programas realizados en acuerdo con el gobierno, así como en asociaciones civiles, reportan mayores resultados porque el involucramiento de la población genera y crea un sentido de pertenencia y apoyo.

Aseveran, Escoto y Castro (2015), lo más importante es el involucramiento de todos por una causa común, para que se cumpla el dicho "la unión hace la fuerza". La experiencia en este sentido ha sido fructífera, y se ha podido palpar como en cada comunidad, cada persona, organización, asociaciones, entre otros, hacen aportes valiosos que permiten lograr los objetivos y metas planeados. En este orden de ideas, para estos autores, los programas de responsabilidad social externa se caracterizan, regularmente, por tener en común los siguientes aspectos:

- Misión: crear cambios conductuales en los miembros de la comunidad donde operan las empresas, incidiendo en el desarrollo social, cívico, económico y cultural.

- Visión: convertir una comunidad subdesarrollada en un ejemplo de productividad y civismo.

- Estrategia: crear programas de responsabilidad social externa, con la participación activa de los residentes de la comunidad para lograr el desarrollo personal y comunitario, permitiéndoles ser entes productivos con sostenibilidad en el tiempo.

En este marco referencial, estos autores afirman, los programas de responsabilidad social externa pueden ser realizados en diferentes vertientes. Regularmente, dependen del foco de la empresa. Los enfoques más comunes son en las áreas de desarrollo social y desarrollo económico.

Con base a lo expuesto, se considera que, en las operaciones del banco occidental de descuento del municipio Lagunillas-Venezuela los programas de responsabilidad social externa deben ser vistos como los medios de implementación o de puesta en práctica de los principios y valores de la responsabilidad social con que cuentan las mismas para el cumplimiento de ésta. Para su análisis se aborda el criterio de Escoto y Castro (2015), asumiéndose como programas de responsabilidad social externa los referidos al desarrollo social y desarrollo económico. 


\section{Desarrollo social}

Una empresa sostenible es aquella que integra valores de desarrollo social, la acción social entendida como una de las formas de que dispone la empresa para plasmar sus compromisos con la comunidad local, según lo refiere Fernández (2009). La concepción más extendida, de lo que se entiende por desarrollo social, es la dedicación de recursos empresariales a proyectos que tienen que ver con el apoyo a personas desfavorecidas, y esos es parte de lo que se entiende por responsabilidad de las empresas.

Al respecto, Fernández (2009), señala que, los recursos dedicados suelen ser de diversos tipos (productos y servicios, capital humano, empleo, financiación, entre otros), y se destinan principalmente a servicios sociales, salud, educación, formación profesional y empleo.

Al mismo tiempo se afirma que, existe otra visión, también desde el mundo empresarial, que distingue tres grandes vectores dentro de la acción social: El primero, recoge todo aquello que son patrocinios de aquellas cosas que las administraciones públicas no pueden llegar. Por ejemplo, todo lo que son actividades culturales, es decir, que van desde la música, el teatro, la pintura, bellas artes, entre otros.

El segundo, incluye temas puros de acción social, como puede ser la cooperación con organizaciones sociales, ONG, entre otras, aportando a lo social algunos excedentes que pueden ser absolutamente complementarios a las actividades de ONG y las administraciones. El tercero, agrupa aquellos temas que pueden hacer que la vida sea por un lado más eficiente para las compañías, pero desde luego más satisfactoria para las personas, como, por ejemplo, la compatibilización de vida personal con vida laboral, con carrera profesional, entre otras.

Ahora bien, la definición de responsabilidad social externa de la Comisión Europea (2014), incide en la idea de que es una integración de las operaciones comerciales y las relaciones con los interlocutores, poniendo la responsabilidad social en el núcleo de la empresa con incidencia en la acción periférica y social de ésta hacia terceros.

Desde esta perspectiva, afirman Escoto y Castro (2015), la responsabilidad social externa va más allá de la pura filantropía o de la acción social por la cual la empresa revierte en la sociedad, de forma más o menos generosa, parte de los beneficios procedentes de su actividad en el mercado. La acción social no implica una transformación profunda de la empresa, que sí es obligada en los procesos de cambio hacia una empresa socialmente responsable.

En este sentido, a criterio de Abab (2010), la acción social de la empresa se materializa en proyectos que se pueden clasificar en función de los tipos de recursos aplicados: programas apoyados en productos y servicios, en colaboración con empleados, de integración laboral, de patrocinio y financiación o corporativos, Una acción social avanzada integra distintos tipos de programas. Afirma este autor que, según el Informe 2014 del Observatorio de la Acción Social de la Empresa en España que recoge información de 140 empresas, el 40 por ciento poseía un 
planteamiento de la acción social basado en la filantropía, el 51 por ciento hacía hincapié en la comunicación, con el objetivo de generar publicidad positiva o motivación interna.

El resto se encontraba en una fase en la cual su acción social ya era un factor de competitividad tras varios años de trabajo, y les había ayudado a conseguir resultados relevantes en el ámbito del empleo de personas desfavorecidas, participación de su personal, aportación de servicios de valor añadido a proyectos sociales o contribución al desarrollo local. Se trataba de acciones y planteamientos enfocados al medio plazo, nada vinculados con las campañas de marketing social de carácter coyuntural, y que no suele apreciar tanto el ciudadano.

Con base a esto, Abad (2010) hace la siguiente reflexión:

la acción social del futuro no será ni filantropía ni marketing, que se considerarán fases previas. Alcanzará su madurez cuando la empresa descubra que cabe un enfoque más estratégico, cuando la adecuada integración de lo económico y lo social signifique una ventaja competitiva, se plantee como una inversión en vez de un donativo, y potencie el trabajo en equipo frente al individual. Así lo entienden ya las empresas mejor percibidas por su acción social, que utilizan de manera inteligente su capacidad de innovación, su espíritu emprendedor y su red de relaciones para alinear su estrategia empresarial y la posibilidad de mejorar su entorno social (s/p).

En resumen, en cuanto a la posición de los autores citados, se considera el desarrollo social como uno de los programas de implementación de la responsabilidad social externa de la banca, el cual se circunscribe en una manera de concretar el compromiso empresarial con la comunidad local en la que se inserta la organización.

Se entiende entonces que la responsabilidad social cuenta, entre los componentes de su dimensión externa, con la integración de los bancos en su entorno local. Aunque los límites de este entorno puedan ser algo difusos, parece obvio que el grupo de interés que aquí entra en juego son comunidades directamente afectadas por las operaciones de una empresa.

\section{Desarrollo económico}

Desde la perspectiva económica, la responsabilidad social externa de las empresas tiene como misión la maximización de valor para sus grupos de interés. Al respecto, para Fernández (2009), sólo las empresas competitivas y generadoras de beneficios son capaces de contribuir a largo plazo al desarrollo sostenible, creando riqueza y empleo sin poner en peligro las necesidades sociales y medio ambientales de la sociedad. De hecho, únicamente las empresas rentables son sostenibles y tienen capacidad de llevar a cabo prácticas socialmente responsables.

Tradicionalmente esta responsabilidad, se ha traducido en la búsqueda del máximo beneficio y máximo valor para el accionista, así como también una óptima eficiencia y productividad. En otras palabras, maximizar beneficios y la producción con los recursos de los que se disponen. Para ello se 
compara el coste de la utilización de unos recursos con la obtención de unos resultados. En los costos suele incluirse el costo de materias primas, energía, recursos financieros, laborales, entre otros; y en los resultados los ingresos como medida de los mismos.

Un comportamiento responsable social de la empresa, implicaría desde el punto de vista económico crear valor: para el cliente, atendiendo a sus demandas ofreciendo precios competitivos, bienes y servicios de calidad (atención de quejas, consultas, entre otros); para los proveedores, pagando precios justos por sus productos o servicios y sin abusar del poder de mercado; para la sociedad en su conjunto preservando y creando empleo y teniendo un comportamiento medio ambiental sostenible, según la opinión de Fernández (2010).

Por su parte, Gómez (2013) asegura, desde una perspectiva económica todas las empresas deben ser responsables de sus grupos de interés, quienes se interesan en procedimientos contables apropiados, información relevante acerca de su desempeño actual y proyectado, y desde luego, recuperar sus inversiones. El desarrollo económico afecta tanto a sus empleados como a la comunidad en general, por una sencilla razón, y es que sin ellos es probable que la empresa no sobreviva lo suficiente para participar en actividades de responsabilidad social.

En este marco, según De la Cuesta (2010), en los objetivos de la responsabilidad social empresarial y el desarrollo sostenible se incluyen el prescindir del compromiso de las empresas con sus entornos locales, así como su relación con otras comunidades, incluyendo actividades de cooperación al desarrollo económico. Las empresas han de comprometerse con la rendición de cuentas, propiciando la mejora constante de sus sistemas de gestión interna y verificación de resultados en la esfera económica.

En función de los postulados expuestos, se plantea que, el desarrollo económico se genera directamente de los ingresos y de las operaciones bancarias, y ejerce su influencia de manera directa a través de los programas de la responsabilidad social externa, garantizándole el éxito y el poder sostenerse, siendo perdurable en el tiempo. Sólo los bancos competitivos y generadores de beneficios son capaces de contribuir a largo plazo al desarrollo sostenible, creando riqueza y empleo sin poner en peligro las necesidades sociales y medio ambientales de la sociedad.

\section{MÉTODO}

$\mathrm{L}$ a investigación fue de tipo descriptiva, con diseño no experimental, de campo y transeccional. La población estuvo conformada por los gerentes, subgerentes y ejecutivos de ventas del Banco Occidental de Descuento del municipio Lagunillas, específicamente dos ubicados en Ciudad Ojeda y uno en Lagunillas, los cuales son un total de veintisiete (27), tal como se refleja en la tabla 1. 
Tabla 1. Distribución de la población

\begin{tabular}{llll}
\hline Banco Occidental de Descuento & Gerentes & Subgerentes & Ejecutivos de Ventas \\
\hline BOD Ciudad Ojeda Av. Intercomunal & 1 & 2 & 7 \\
BOD Ciudad Ojeda Casco Central & 1 & 2 & 7 \\
BOD Lagunillas & 1 & 1 & 5 \\
Total & $\mathbf{3}$ & $\mathbf{5}$ & $\mathbf{1 9}$ \\
\hline
\end{tabular}

La recolección de la información se llevó a cabo a través de un cuestionario autoadministrada conformado por 8 ítems con un escalamiento de frecuencia con tres alternativas de respuestas, cuyas categorías fueron: siempre (3); algunas veces (2) y nunca (1). El cuestionario cual fue validado por el juicio de cinco expertos y obtuvo un 0,913 de confiabilidad según el coeficiente Alfa Cronbach.

La información fue procesada a través de la estadística descriptiva específicamente mediante la media aritmética, para la cual se diseñó un baremo de interpretación, reflejado en la tabla 2.

Tabla 2. Categoría de análisis para la interpretación del promedio

\begin{tabular}{llll}
\hline Alternativas & Opción de respuesta & Rango para la media & Nivel de respuesta para la variable \\
\hline 3 & Siempre(S) & $2.35 \leq 3.00$ & Alta presencia/Fortaleza \\
2 & Algunas Veces (AV) & $1.68 \leq 2.34$ & Mediana presencia/Debilidad \\
1 & Nunca(N) & $1.00 \leq 1.67$ & Baja presencia/Debilidad \\
\hline
\end{tabular}

\section{RESULTADOS}

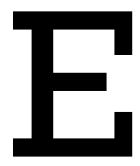

n la tabla 3 se resumen los resultados para la dimensión, con una media de 2,37, implicando alta presencia de los programas en la RSE que realizan en las operaciones de los bancos bajo estudio, delineándola como fortalezas. De manera específica, se pudo evidenciar alta presencia del desarrollo económico (2,57), constituyéndolo en una fortaleza de su RSE; y mediana presencia del desarrollo social $(2,17)$ delineándolo como una debilidad, e indicando que se requieren mejoras en este aspecto.

Tabla 3. Dimensión: Programas de responsabilidad social externa

\begin{tabular}{lll}
\hline INDICADORES & MEDIA & CATEGORÍA \\
\hline Desarrollo social & 2,17 & Mediana presencia/debilidad \\
Desarrollo económico & 2,57 & Alta presencia/fortaleza \\
DIMENSIÓN & $\mathbf{2 , 3 7}$ & Alta presencia/fortaleza \\
\hline
\end{tabular}


Estos resultados, tienen congruencia con lo enunciado por Baltera y Díaz (2012) para quienes, las prácticas de la empresa socialmente responsable reflejan el compromiso a hacer negocios de forma que preserve o aumente, en lugar de dañar, beneficios a las comunidades cercanas, trabajadores, medio ambiente y la conducta económica justa. Sin embargo, no basta con el sólo compromiso, este proceso debe ir acompañado de mecanismos claros de implementación o puesta en práctica de los principios y valores de la responsabilidad social, a través de programas de responsabilidad externa, incluyendo seguimiento y verificación independientes.

De esta forma, las operaciones del banco occidental de descuento del municipio LagunillasVenezuela, los programas de responsabilidad social externa son vistos como los medios de implementación o de puesta en práctica de los principios y valores de la responsabilidad social con que cuentan las mismas para el cumplimiento de ésta, asumiéndose como programas de responsabilidad social externa los referidos al desarrollo social y desarrollo económico.

En lo concerniente al detalle de cada uno de los indicadores utilizados para medir la dimensión, de seguido se especifican los resultados obtenidos al respecto, mostrados en la tabla 4, la cual recoge el resultado de la dimensión y cada uno de sus indicadores. De esta forma, se evidencia mediana presencia para el desarrollo social con una media de 2,17 , siendo esto una debilidad de su RSE.

En específico, delinearon con mediana presencia las actividades medidas en este indicador referidas a si: destinan recursos a servicios sociales (educación, salud, empleo, cultura, formación profesional) (2,19); realizan patrocinios de actividades culturales (música, teatro, pintura, bellas artes, entre otros) $(2,11)$; y aportan a la sociedad algunos excedentes económicos que pueden ser absolutamente complementarios a las actividades de las ONG $(1,89)$; señalándolas como debilidades de su RSE, indicando oportunidades de mejora al respecto.

Lo anterior, valida medianamente el postulado de Fernández (2009), para quien, una empresa sostenible es aquella que integra valores de desarrollo social; la acción social entendida como una de las formas de las cuales dispone la empresa para plasmar sus compromisos con la comunidad local. La concepción más extendida, de lo que se entiende por desarrollo social, es la dedicación de recursos empresariales a proyectos que tienen que ver con el apoyo a personas desfavorecidas, y esos es parte de lo que se entiende por responsabilidad de las empresas.

De igual manera, medianamente se valida el criterio de la investigadora, al considerar que el desarrollo social es uno de los programas que se circunscribe en una manera de concretar el compromiso empresarial con la comunidad local en la que se inserta la organización. 
Tabla 4. Indicadores: Programas de responsabilidad social externa

\section{INDICADOR: DESARROLLO SOCIAL}

N. ${ }^{\circ}$ ITEMS

1

Destinan recursos a servicios sociales (educación, salud, 2,19 empleo, cultura, formación profesional).

2

Realizan patrocinios de actividades culturales (música, 2,11 teatro, pintura, bellas artes, entre otros).

3

Aportan a la sociedad algunos excedentes económicos que pueden ser absolutamente complementarios a las actividades de las ONG

4 Consideran su acción social como un factor de 2,48 competitividad.

\section{INDICADOR 2,17}

\section{CATEGORIA}

Mediana presencia

Debilidad

Mediana presencia

Debilidad

1,89 Mediana presencia

Debilidad

Alta presencia/Fortaleza

\section{Mediana presencia/Debilidad}

Alta presencia/Fortaleza

Alta presencia/Fortaleza

Alta presencia/Fortaleza justos por sus productos o servicios sin abusar del poder de mercado.

8 Crean valor para la sociedad en conjunto teniendo un 2,30 comportamiento medioambiental sostenible.

Mediana presencia

Debilidad

\section{INDICADOR 2,57 Alta presencia/Fortaleza}

En cuanto al programa de desarrollo económico, se observa alta presencia con una media de 2,57; siendo esto una fortaleza para su RSE. No obstante, ofrecen una oportunidad de mejora al calificar con mediana presencia el que: crean valor para la sociedad en conjunto teniendo un comportamiento medioambiental sostenible $(2,30)$, signándola como una debilidad.

Este resultado de alta presencia del programa de desarrollo económico, valida a Gómez (2013) cuando asegura, desde una perspectiva económica todas las empresas deben ser responsables de sus grupos de interés, quienes se interesan en procedimientos contables apropiados, información relevante acerca del desempeño actual y proyectado y, desde luego, recuperar sus inversiones. El desarrollo económico afecta tanto a sus empleados como a la comunidad en general, por una sencilla razón, y es que sin ellos es probable que la empresa no sobreviva lo suficiente para participar en actividades de responsabilidad social. 
Asimismo, existe alta congruencia con lo expuesto por la investigadora, al referir que el desarrollo económico genera directamente ingresos de las operaciones bancarias, y ejerce su influencia de manera directa a través de los programas de la responsabilidad social externa, garantizándole el éxito y el poder sostenerse, siendo perdurable en el tiempo.

En términos generales, los programas de la RSE, pueden ser realizados en diferentes vertientes; y regularmente dependen del foco de las operaciones del banco occidental de descuento del municipio Lagunillas-Venezuela; y los enfoques más comunes son en desarrollo social y desarrollo económico.

\section{CONCLUSIONES}

$\mathrm{E}$ n lo que concierne al objetivo, en el cual se identificaron los programas de responsabilidad social externa presentes en las operaciones del banco occidental de descuento del municipio Lagunillas-Venezuela, siendo ellos: desarrollo social y desarrollo económico; se concluye que, en las operaciones del banco analizado los programas de RSE estudiados poseen alta presencia, donde el desarrollo económico alcanza alta presencia, y el desarrollo social tiene mediana presencia, observándose posibilidades de mejora.

Al respecto, aun cuando se alcanza alta presencia de estos programas, se conoció de oportunidades de mejora en cada uno de los indicadores medidos, así las cosas, para el desarrollo social es importante garantizar que se destinen recursos a servicios sociales y velar porque se aporten a la sociedad algunos excedentes económicos que pueden ser absolutamente complementarios a las actividades de las ONG.

En cuanto al desarrollo económico se hace imprescindible garantizar la creación de valor para la sociedad en conjunto, teniendo un comportamiento medioambiental sostenible.

\section{REFERENCIAS}

Abad, F. (2010). Acción social en la empresa, algo más que una moda. Disponible en: http://www.redtelework.com/imprimir.aspx?id=13 744

Añez, S.; Hernández, R.; Silvestri, K., y Gómez, O. (2008). Análisis de los elementos que conforman la responsabilidad social corporativa. Revista Formación Gerencial. Año 7 Número 2 p. 173 185

Baltera, P., y Díaz, E. (2012). Responsabilidad social empresarial. Alcances y potencialidades en materia laboral. Cuaderno de Investigación $\mathrm{N}^{\circ} 25$.
Gobierno de Chile. Dirección del Trabajo Departamento de Estudios. Chile

Comisión Europea (2014). La responsabilidad social de las empresas (RSE) en la UE. Disponible en: http://ec.europa.eu/social/main.jsp?catld=331\&lan gld $=e s$

De la Cuesta, M. (2010). Responsabilidad Social. Editorial Consello Social. Universidad de la Coruña. España 
Escoto, A., y Castro, E. (2015). Guía de responsabilidad social empresarial. INFOTEP. Certificación ISO 9001. Primera edición, noviembre de 2015. República Dominicana

Fernández, R. (2009). Responsabilidad social corporativa. Nueva cultura empresarial. Editorial Club Universitario. España

Fernández, R. (2010). Dimensiones de la responsabilidad social empresarial. Sus actores. Papel de las políticas públicas. Diario responsable. La RSE Global. Disponible en: http://diarioresponsable.com/opinion/11467dimensiones-de-la-responsabilidad-socialempresarial-sus-actores-papel-de-las-politicaspublicas
Gómez, H. (2013). Empresa internacionalizada y responsabilidad social: Un matrimonio convencido. Cuadernos Básicos. Publicaciones ICEX. España

Libro Verde de la Comunidad Económica Europea (2001). Disponible en: http://eurlex.europa.eu/legalcontent/ES/TXT/?uri=CELEX\%3A52001DC0366

Martínez, H. (2011). Responsabilidad social y ética empresarial. 1era edición. Ediciones ECOE. Bogotá. Colombia

Navarro, F. (2012). Responsabilidad Social Corporativa: Teoría y práctica. ESIC Editorial. Segunda Edición. Madrid. España 